Published in final edited form as:

Nat Methods. 2019 March ; 16(3): 235-238. doi:10.1038/s41592-019-0327-9.

\title{
Universal Light-Sheet Generation with Field Synthesis
}

\author{
Bo-Jui Chang ${ }^{1,{ }^{*}}$, Mark Kittisopikul ${ }^{2,4,}{ }^{*}$, Kevin M. Dean ${ }^{1}$, Philippe Roudot ${ }^{1,3}$, Erik S. Welf ${ }^{1,3}$, \\ and Reto Fiolka ${ }^{1,3}$ \\ ${ }^{1}$ Department of Cell Biology, UT Southwestern Medical Center, Dallas, TX, USA. \\ 2Department of Biophysics, UT Southwestern Medical Center, Dallas, TX, USA. \\ ${ }^{3}$ Lyda Hill Department of Bioinformatics, UT Southwestern Medical Center, Dallas, TX, USA. \\ ${ }^{4}$ Department of Cell and Molecular Biology, Feinberg School of Medicine, Northwestern \\ University, Chicago, IL, USA.
}

\section{Abstract}

We introduce Field Synthesis, a theorem that can be used to synthesize any scanned or dithered light-sheet, including those used in lattice light-sheet microscopy (LLSM), from an incoherent superposition of one-dimensional intensity distributions. This user-friendly and modular approach offers a drastically simplified optical design, higher light-throughput, simultaneous multicolor illumination, and a $100 \%$ spatial duty cycle that provides biological imaging with low rates of photobleaching.

\begin{abstract}
For years, confocal microscopy was the method of choice for subcellular volumetric imaging of biological specimens. ${ }^{1}$ However, the exceedingly small dwell-times of modern confocal microscopes (e.g., $<2$ microseconds) necessitates high peak illumination intensities, which in addition to the excitation wavelength, accelerates photobleaching and phototoxicity. 2,3 Further, regions above and below the laser focus are unnecessarily illuminated, resulting in the expenditure of precious photons that do not productively contribute to image formation. In contrast, light-sheet fluorescence microscopy (LSFM) adopts an orthogonal imaging geometry whereby the sample is illuminated from the side and fluorescence is collected at 90 degrees with high numerical aperture optics and million-fold camera-based parallelization. ${ }^{4}$ Because fluorescence excitation above and below the depth-of-focus is minimized, optical sectioning is automatically achieved, and unnecessary illumination of the specimen is avoided. Consequently, LSFM permits unparalleled long-term imaging of dynamic biological specimens.
\end{abstract}

\footnotetext{
Users may view, print, copy, and download text and data-mine the content in such documents, for the purposes of academic research, subject always to the full Conditions of use:http://www.nature.com/authors/editorial_policies/license.html\#terms

Correspondence Reto.Fiolka@utsouthwestern.edu.

Author Contributions

R.F., K.M.D., and B-J.C. designed the research. B-J.C. and R.F. designed, built and operated the microscope. R.F., K.M.D., P.R., and B-J.C. performed image analysis. E.W. provided the MV3 cancer cells. M.K. carried out mathematical derivations, proved the theorems, and revised the MATLAB code. R.F., K.M.D., and B.-J.C wrote the manuscript, and all authors read and provided feedback on the final manuscript.

*These authors contributed equally to this work

Competing Interests

R.F., B-J.C. and M.K. have filed a patent for the Field Synthesis process and its applications to microscopy.
} 
In LSFM, a critical tradeoff between axial resolution (influenced by the thickness of the light-sheet), field of view (the length over which a beam can approximate a sheet), illumination duty-cycle, and illumination confinement exists. In an effort to simultaneously optimize these optical parameters, the Betzig laboratory developed lattice light-sheet microscopy (LLSM) ${ }^{5}$ Here, the specimen is coherently illuminated with multiple plane waves that sculpt a propagation-invariant 2-dimensional optical lattice. In its most widely adopted operating mode, the optical lattice is dithered (i.e., scanning it over a small lateral distance, see Supplementary Table 1) to form a time-averaged sheet of light. Unfortunately, LLSM requires an optical train that is complex, expensive, and lossy $(\sim 0.1-1 \%$ input laser power delivered to the sample). ${ }^{5}$

In this manuscript we show that any scanned light-sheet (i.e., a time-averaged light-sheet obtained by rapidly scanning a 2D intensity distribution laterally ${ }^{6}$ ), including dithered lattice light-sheets, can be synthesized by a simple and universal optical process described by a mathematical theorem that we refer to as Field Synthesis (Supplementary Note 1). Applied to Bessel beam light-sheets, Field Synthesis achieves a $100 \%$ spatial duty cycle (e.g., the entire field of view is illuminated simultaneously during the exposure of the camera) and is therefore more sensitive and results in less photobleaching than time-averaged light-sheets generated by lateral beam scanning. Applied to the most commonly used dithered mode of LLSM, light-losses in the excitation train are reduced 15-fold (Supplementary Note 2) and simultaneous multi-color imaging becomes possible. Furthermore, the number of optical components is halved. Importantly, no spatial light-modulator, diffraction optics or polarization optics are needed, which significantly reduces the complexity and cost of a LLSM system (see Figure S1 and Supplementary Note 2). We mathematically prove the Field Synthesis theorem, demonstrate proof-of-concept imaging, and provide software and documentation so that the biological community may adopt the technology.

The essence of the Field Synthesis theorem can be described as follows: In the ideal line scan case, the projection of the squared modulus of a two-dimensional function is equivalent to the average of the squared modulus of a line scan in the Fourier domain of the same function (Supplementary Notes 1 and 3, Movie S1 and S2). Here, the line scan follows the same direction in Fourier space as the projection in real space (e.g., $\mathrm{k}_{\mathrm{x}}$ and $\mathrm{x}$, respectively). Importantly, this has practical applications to scanned light-sheets, where a time-averaged light-sheet is obtained by laterally scanning the illumination beam. In a conventional LSFM, the illumination beam is shaped by light distribution with spatially varying amplitude and phase in the back-pupil plane of the excitation objective, which can be described as 2dimensional pupil function (Figure 1A, Top Row). ${ }^{7}$ Our theorem predicts that if a focused line is scanned over the same pupil function, an identical time-averaged light-sheet is obtained (Figure 1A, Bottom Row). We also provide a generalized theorem describing nonideal line scans with an arbitrary line profile (Supplementary Note 1). Thus, by simply scanning a focused line over an appropriate pupil mask (e.g., a 2-dimensional spatial filter), we can create any type of scanned or dithered light-sheet, including those used by LLSM.

Unlike other attempts to simplify scanned Bessel beam light-sheets or LLSM, ${ }^{8}$ Field Synthesis is expected to reproduce the time-averaged excitation intensity distribution of these methods exactly. Hence axial resolution, out-of-focus illumination and field of view 
can be tailored to the same degree as with the underlying original light-sheet techniques. While the time-averaged intensity distributions obtained by Field Synthesis are predicted to be identical to dithered or scanned light-sheets, there are important differences: (i) The spatial duty cycle is $100 \%$ for symmetric pupil functions (as used in Bessel and LLSM) since the light-sheet is synthesized from a series of cosines (which have maxima at their origin). (ii) Field Synthesis, as implemented here, is based on refraction and hence can be implemented with achromatic optics, permitting simultaneous multi-color excitation.

To evaluate this optical principle, we compared the three-dimensional intensity distributions of Bessel and LLSM with those generated by Field Synthesis (see Supplementary Information for details). As seen in Figures 1B-G, both in cross-sectional views as well as in the propagation direction, field synthesis produces identical light-sheets. Interestingly, for LLSM, it has been hypothesized that complex 2D interference patterns are necessary for its advantageous properties, such as aspect ratio of the sheet and suppression of its sidelobes. Remarkably, we found that the exact same light-sheets can be obtained by incoherently summing 1D intensity patterns (see Supplementary Notes 1 and 3, Figures S2-7, and Supplemental MATLAB code), even in the presence of non-ideal line scans (Figure S8).

To compare the performance between a Bessel beam LSFM constructed using conventional beam scanning and Field Synthesis, we measured photobleaching curves of $100 \mathrm{~nm}$ fluorescent microspheres and microtubule Plus-End Tracking Proteins (labeled with EB3mNeonGreen) in U2OS cells. In both cases, owing to its two orders of magnitude higher duty cycle, Field Synthesis resulted in lower rates of photobleaching (Figures 2A-C, Movie S3). The low spatial duty cycle of scanned Bessel beams has been previously identified to be the cause for increased photo-bleaching and spurred the development of LLSM. ${ }^{5,9,10}$ Our results show a new, general mechanism to improve spatial duty cycle for propagation invariant beams without requiring complex $2 \mathrm{D}$ interference patterns and retaining the original light-sheet. ${ }^{11-14}$ It has to be noted that Field Synthesis cannot be combined with virtual confocal line detection in scanned light-sheets, ${ }^{15-17}$ as the entire field of view is illuminated instantaneously.

Compared to LLSM, Field Synthesis improves the spatial duty cycle theoretically by a factor of two (estimated via numerical simulations of the intensity distribution in the main lobe of the light-sheet). Practically, our measurements indicate that Field Synthesis matches the excellent low photobleaching of a dithered square lattice (Figure S9) and provides comparable biological imaging performance (Figure S10) while offering other advantages such as simultaneous multicolor imaging. To demonstrate that Field Synthesis can be used as an alternative to LLSM, we performed sensitive dual-color imaging of MV3 melanoma cells labeled with a PI3K biosensor (GFP-AktPH) and a membrane-localized fluorescent protein (Td-tomato-CAAX). Both conventional LLSM and Field Synthesis LLSM allowed us to resolve fine cellular features such as small membrane protrusions and filopodia and provided excellent optical sectioning. However, because Field Synthesis is compatible with simultaneous multicolor imaging, the volumetric imaging rate is two-fold faster than conventional LLSM if two-channels are acquired, despite using identical imaging parameters (camera integration time, z-step size, etc.). This allowed us to observe rapid subcellular events such as small membrane protrusions and filopodia dynamics, which were 
temporally undersampled in the sequential acquisition mode afforded by conventional LLSM (Figure 2D-K, Movie S4-S5). While sequential LLSM acquisition could in principle be accelerated by using shorter camera integration times, this would come at the expense of loss of signal or higher peak illumination intensities and might not be possible due to camera bandwidth limitations. Thus we believe that simultaneous multicolor imaging with Field Synthesis expands the capabilities of LLSM, particularly in spectral imaging applications. ${ }^{18}$

In conclusion, we have introduced with the Field Synthesis theorem a novel and universal mechanism to generate scanned and dithered light-sheets. Importantly, Field Synthesis drastically simplifies the optical train compared to lattice light-sheet microscopy. The consequence is that there are less critical components to align and temporally control, which eases building and operating such an instrument, both practically and financially.

Additionally, simultaneous multicolor acquisition can be achieved. Field synthesis was not designed to allow structured illumination LLSM through a single objective, ${ }^{5}$ but is conceptually compatible with structured illumination using multiple objectives. ${ }^{19}$ In our view, such multi-objective illumination will allow to unlock the full potential of structured illumination microscopy with light-sheets, and we believe that Field Synthesis will be more experimentally tractable than a conventional lattice setup for such a configuration.

Beyond LLSM, the field synthesis principle applies to any type of intensity distribution. So, by simply changing the pupil filter, other light-sheet types can be generated, including, Bessel, Gaussian, or Airy light-sheets. Furthermore, our insight that any light-sheet can be synthesized by a superposition of one-dimensional functions also implies that field synthesis could be used to rationally design new forms of light-sheets, each uniquely optimized to specific merit functions. Thus, we believe that Field Synthesis will make light-sheet fluorescence microscopy modular and democratize advanced techniques, including but not limited to lattice light-sheet microscopy.

\section{Online Methods.}

\section{Microscope Setup}

The basic units to generate lattice and field synthesis light-sheets are shown in Figure S1 and our microscope setup is illustrated in Figure S11. A 488 (Sapphire 488-300 LP, Coherent) and a $561 \mathrm{~nm}$ (OBIS 561-150LS, Coherent) are combined with a dichroic mirror (LM01503-25, Semrock), and focused through a 50-micron spatial filter (P25H, ThorLabs) with a $50 \mathrm{~mm}$ focal length achromatic lens (AC254-050-A, Thorlabs). Thereafter, flip mirror 1 is used to select either the LLSM or Field Synthesis optical paths, and the beam is recollimated with a 75 (AC254-075-A, Thorlabs) or 400 (AC508-400-A, Thorlabs) mm focal length achromatic lens, respectively. Further, the optical system is operated in a fluorescence (e.g., with orthogonal detection, Figure S11A) or transmission (e.g., with diascopic detection, Figure S11B) mode, to image fluorescent specimens and investigate light-sheet properties, respectively.

The LLSM path is a simplified version of the original set-up. ${ }^{1}$ The collimated and expanded beam is further magnified and shaped to a line beam by a pair of achromatic cylindrical lenses (68-160, Edmund optics and ACY254-200-A, Thorlabs). The line beam uniformly 
illuminates a region on the spatial light modulator (SLM, SXGA-3DM, Forth Dimension Displays) where the lattice pattern is displayed. A polarized beamsplitter (10FC16PB.3, Newport) and a half-wave plate (AHWP10M-600, Thorlabs) are placed in front of the SLM, and together with the SLM form the pattern generator. ${ }^{5,20}$ The lasers were assembled in a way that the polarization states fit the pattern generator. The diffraction light from the SLM is focused by an achromatic lens (AC508-400-A, Thorlabs) on a custom-designed mask (mask 1, Photo Sciences, Inc.). The mask has various annuli to block unwanted diffraction orders (mainly the $0^{\text {th }}$ order but also higher orders). Different sizes of the annuli correspond to different outer and inner NAs on the back focal plane of the illumination objective lens. After passing through the mask, the desired diffraction orders are de-magnified through two achromatic lenses (AC254-150-A, AC254-100-A, Thorlabs) and fit into the size of a galvanometer (Galvo mirror, 6215H, Cambridge Technology). A folding mirror, an achromatic lens (AC-254-100-A, Thorlabs), the flip mirror 2 and a tube lens (ITL-200, Thorlabs) then direct the light to the illumination objective lens (40X, NA0.8, 3.5mm WD, CFI Apo 40XW NIR, Nikon) to form a light-sheet in sample space. All distances between a pair of lenses equal to the summation of their focal lengths so any pair of lenses forms a $4 \mathrm{f}$ arrangement. Thus, The SLM is conjugated to the focal plane of the illumination objective lens. The mask is at the Fourier plane of the SLM and is conjugated to the back focal plane of the objective lens. As the galvanometer is conjugated to the mask and the back focal plane of the objective lens, it scans the light-sheet laterally (y) on the focal plane of the detection objective, i.e. enables rapid lateral scanning of a Gaussian or Bessel light-sheet and the dithered mode of the lattice light-sheet. Figure S7 shows typical lattice interference patterns that we could obtain with our setup.

The Field synthesis path consists of an iris (IDA20, Thorlabs), an achromatic cylindrical lens (ACY254-050-A, Thorlabs), an achromatic lens (AC254-075-A, Thorlabs), another customdesigned mask (mask 2), and a tube lens (ITL-200, Thorlabs). An alternative beam expander design to illuminate efficiently smaller field of views is discussed in Supplementary Note 4 and Figure S12. A galvanometer (Galvo mirror, 6215H, Cambridge Technology) and a folding mirror are placed in between the achromatic cylindrical lens and the achromatic lens. The Galvo mirror is conjugated to the sample plane, which is a similar configuration as used in mSPIM. ${ }^{21}$ This path shares the same final tube lens and illumination objective lens with the lattice LSFM path. All distances between a pair of lenses equal to the summation of their focal lengths so any pair of lenses forms a $4 \mathrm{f}$ arrangement. The galvanometer in the Field Synthesis path is conjugated to the focal plane of the illumination objective lens. The mask is conjugated to the back focal plane of the objective lens, which is conjugated to the same plane as the mask in the Lattice LSFM path. Since the magnification between the mask and the back focal plane of the objective lens is different in the Field Synthesis LSFM path and the Lattice LSFM path, the sizes of the annuli on mask 2 are designed to match the outer and inner NAs resulted from the annuli on mask 1, as shown in Supplementary Table 2.

The detection path is orthogonal to the illumination path as in a conventional LSFM set-up and it consists of an detection objective lens (40X, NA0.8, 3.5mm WD, CFI Apo 40XW NIR, Nikon), a tube lens (ITL-200, Thorlabs), a dichroic mirror (Di03-R561-t3-25×36, Semrock) that separates the green and the red fluorescence, and two identical sCMOS cameras (Orca Flash4.0 v2, Hamamatsu). A red fluorescence emission filter (FF01-593/ 
LP-25, Semrock) and a green fluorescence emission filter (ET525/50m, Chroma) is placed in front of camera 1 and camera 2, respectively. A piezo stage (P621.1CD, Physik Instrumente) is used to move the sample diagonally to the detection axis in the fluorescence mode (Figure S11A) or move the detection objective lens in the transmission mode (Figure S11B).

In the transmission mode (Figure S11B), the detection objective lens is placed along the propagation of the light-sheet and an achromatic lens (AC508-400-A, Thorlabs) is used as the tube lens. An ND filter (ND40A, Thorlabs) is placed in front of the sCMOS camera (Orca Flash4.0 v2, Hamamatsu) to reduce the intensity of the straight light from the lasers. The combination of the $400 \mathrm{~mm}$ tube lens and the 40x objective lens offers a final magnification of $80 \mathrm{x}$, which results in one pixel on the camera corresponds to $81.25 \mathrm{~nm}$ and that can present the light-sheet with a reasonable contrast and resolution. By stepping the detection objective lens with the piezo stage through the illumination lattice, we investigate and measure the $3 \mathrm{D}$ light-sheet properties.

The pattern on the back focal plane of the excitation objective lens can be observed by Camera 4 (DCC1545M, Thorlabs) with an ND filter (ND10A or ND20A, Thorlabs) in the front when flipped mirror 3 is used. Two achromatic lenses (AC254-100-A and AC254050-A, Thorlabs) are used to image the back focal plane of the objective lens, which is conjugated to the plane of the masks, on the camera. By observing the pattern on this plane allows us to verify the final outer and inner NAs from both masks and adjust the scan range of the galvo in Field Synthesis.

\section{Microscope control and image acquisition}

The data acquisition computer was equipped with an Intel Xeon E5-2687W v3 processor operating at $3.1 \mathrm{GHz}$ with 10 cores and 20 threads, $128 \mathrm{~GB}$ of $2133 \mathrm{MHz}$ DDR4 RAM, and an integrated Intel AHCI chipset controlling $4 \times 512$ GB SSDs in a RAID0 configuration. All software was developed using a 64-bit version of LabView 2016 equipped with the LabView Run-Time Engine, Vision Development Module, Vision Run-Time Module and all appropriate device drivers, including NI-RIO Drivers (National Instruments). Software communicated with the camera (Flash 4.0, Hamamatsu) via the DCAM-API for the Active Silicon Firebird frame-grabber and delivered a series of deterministic TTL triggers with a field programmable gate array (PCIe 7852R, National Instruments). These triggers included analog outputs for control of mirror galvanometers, piezoelectric actuators, laser modulation and blanking, camera fire and external trigger, and triggering of the ferroelectric spatial light modulator. A timing diagram for the galvanometric beams scanning in Field Synthesis illustrated in Figure S13. All images were saved in the OME-TIFF format. ${ }^{22}$

Some of the core functions and routines in the microscope control software are licensed under a material transfer agreement from Howard Hughes Medical Institute, Janelia Farm Research Campus. The control software code for Field Synthesis can be requested from the corresponding authors and will be distributed in accordance with this material transfer agreement. 


\section{Data Analysis}

Image analysis was performed with Fiji ${ }^{3}$ and MATLAB (Mathworks). To evaluate the rate of photobleaching for $100 \mathrm{~nm}$ fluorescent microspheres, the average intensity of 11 similarly bright beads were measured through time by recording their peak intensity at every timepoint. To this end, a MATLAB script was written that detected the peak intensity of all beads in the dataset and a subset was manually selected that yielded the same average initial intensity for both imaging modalities. The same script was used to initially calibrate the power levels that yielded similar fluorescent counts when the same sample region was imaged with either Bessel beam or Field Synthesis light-sheets. Both the bead imaging as well as the EB3 imaging in the U2OS cells was conducted with such calibrated power levels.

\section{Deconvolution and data post processing}

The point spread function (PSF) used for deconvolution of the lattice light-sheet (conventional and Field Synthesis) data was synthesized by using the PSF Generator plugin in ImageJ. ${ }^{23,} 24$ The Born and Wolf 3D optical model is used to generating the PSF. Illumination and detection PSFs are synthesized in 3D with the practical experimental parameters (i.e. the refractive index, the wavelength, the NA, and the voxel size). The final light-sheet PSF is the product of the illumination and detection PSFs. Since our sample is placed diagonally to the illumination and detection and we scan the sample to acquire the images (sample-scan acquisition), the light-sheet PSF is sheared oppositely referring to the direction of the sample scan. 3D-deconvolution was performed with 10 iterations of the Richardson-Lucy algorithm as implemented in MATLAB. The deconvolved data was sheared at an angle of 45 degrees using an affine transform and bleach corrected with Fiji using the histogram matching method.

For microtubule +TIP data, experimental point-spread functions were obtained by imaging isolated $100 \mathrm{~nm}$ nanospheres with both the conventional Bessel and Field Synthesis modes of microscope operation. We chose experimental PSFs here as the Bessel beam light-sheet has a more complex structure and hence could not be approximated with the numerical PSF Generator. These data were deconvolved, the Z-axis bicubically interpolated so that the voxel dimensions were isotropic $(162.5 \mathrm{~nm})$ and sheared at an angle of 45 degrees using an affine transform. The EB3 data was not bleach corrected.

\section{Biological Sample Preparation}

Both metastatic melanoma (MV3, a kind gift from Dr. Peter Friedl, MD Anderson Cancer Center) and osteosarcoma (U2OS, a kind gift from Dr. Dick McIntosh, University of Colorado Boulder) cell lines were cultured in DMEM supplemented with $10 \%$ fetal bovine serum and penicillin/streptomycin and maintained at 37 degrees Celsius with $5 \% \mathrm{CO}_{2}$ atmosphere. MV3 cells were lentivirally transduced using the pLVX-IRES-PURO and pLVX-IRES-NEO expression systems. The GFP-AktPH probe contains GFP fused to the PH domain of Akt and the td-Tomato membrane marker contains td-Tomato fused to the first 60 base pairs of neuromodulin. U2OS cells were lentivirally transduced with a modified pLVXshRNA2 vector with a truncated CMV promoter to reduce ectopic expression, as previously described. ${ }^{5}$ SK-Mel2 cells (a kind gift from Dr. David Drubin, University of California Berkeley) stably expressing CLC-RFP from their native loci were engineered using Zinc- 
Fingers and TALENS, cultured in DMEM/F12 supplemented with 10\% fetal calf serum at 37 degrees Celsius with $5 \% \mathrm{CO}_{2}$ atmosphere. All cells were plated on $5 \mathrm{~mm}$ coverslips and mounted in a custom sample holder for imaging. ${ }^{6}$

\section{Quantification of Beam Geometry}

For direct measurements of the light-sheet intensity distribution, the excitation light was imaged in transmission with a 40x water-dipping objective, which was scanned in the Ydirection (e.g., along the optical axis) with a 100-micron piezo actuator. Using a $400 \mathrm{~mm}$ tube lens (Figure S11B), the effective pixel size of the imaged beam was $81.25 \mathrm{~nm}$. Beam analysis software, written in MATLAB, identified the laser focus in Y, the size of the beam waist in $\mathrm{Z}$ and the beam propagation length in $\mathrm{Y}$. Since Bessel and lattice light-sheets are propagation invariant (i.e. they maintain their beam profile over extended propagation distances), we did not apply the Rayleigh length criterion to characterize the light-sheets. Instead, we use the full width half maximum in the propagation direction.

\section{Sequential Lattice data}

We developed a simple experiment, which can be reproduced by anyone who has access to a lattice light-sheet microscope, to test the Field synthesis theorem. We termed the procedure sequential lattice: Here, we used the lattice light-sheet illumination path in the square lattice mode and manually positioned a mask to only transmit isolated diffraction orders along the $\mathrm{kz}$ direction (see also Figure S2). This was repeated for three mask positions and the corresponding datasets were added numerically. As the summing of the intensity is incoherent, at no point a 2D lattice pattern emerges, but only one-dimensional intensity patterns are created. Compared to the dithered square lattice, the sequential lattice approach produces the same light-sheets (see Figure S2). In particular, some sidelobe structures are more closely reproduced than in Figure 1D and E. We believe that these small differences arise from slightly different intensity distributions in the pupil plane. Overall, this further confirms that a dithered lattice light-sheet can be synthesized by purely one-dimensional functions, as predicted by the Field Synthesis theorem.

\section{Fluorescent measurement of light-sheet properties}

We have characterized the light-sheets using transmission measurements (Figures 1 and S11B), which provided images of the light-sheets in sample plane. Due to the involved imaging operation, blurring of the observed light-sheet can be expected, but we argue that this effect will equally affect the conventional and Field Synthesis light-sheets. Nevertheless, we also conducted a direct measurement of the light-sheet in sample space. To this end, we mechanically scanned a 50nm fluorescent bead in 0.2 micron steps in the Z-direction and in one-micron steps in the Y-direction through a light-sheet. At every scan position, a widefield image of the fluorescent emission from the bead was taken and the signal on the camera was summed up on a local window surrounding the bead. The window size was chosen such that it contained all diffraction rings of the bead at the largest defocus it was undergoing during the scan. The obtained intensity distributions of a square lattice and a Field Synthesis square lattice light-sheet are shown in Figure S4. The width of the light sheet at its waist was 0.85 microns for the square lattice and 0.91 microns for the Field synthesis light-sheet. In the propagation direction, the Full width half maximum for the square lattice light-sheet was 
16.7 microns and 17.2 microns for the Field Synthesis light-sheet, respectively. These values are in close agreement with the transmission measurements shown in Supplementary Table 3 and the blurring in the transmission measurement appears to be small.

\section{Statistics and Reproducibility}

For statistical analysis, we report the mean, standard deviation, and number of observations. To characterize the Bessel, Lattice, and Field Synthesis light-sheets, each light-sheet was spatially averaged across six different regions for multiple repetitions of the imaging experiment ( $n=2-4$, see Table S3). In the main and Supplementary Figures, only the averaged light-sheet (the mean) is shown, whereas in Supplementary Table 3, the mean and standard deviation of the light-sheet parameters are provided. To further test reproducibility, each Lattice and Field Synthesis light-sheet was imaged eight times in transmission and was automatically processed with a Matlab script. We found similar parameters for beam waist and length to the reported values in this manuscript. The fluorescence-based measurement of the lattice and field synthesis light-sheets was also repeated eight times for each mode. We inspected the data visually and measured beam waist and length and found that the results were similar.

To compare the photobleaching of a digitally scanned Bessel beam and Field Synthesis, the peak intensity of 11 individual beads was evaluated. In Figure $2 \mathrm{C}$, the mean and $95^{\text {th }}$ confidence interval for each time point is shown. Likewise, to compare photobleaching of a Lattice Light-Sheet Microscope and Field Synthesis, the peak intensity of 11 isolated beads was evaluated, and the mean and $95^{\text {th }}$ confidence interval for each time point is shown in Supplementary Figure 9. To compare the effect of photo-bleaching on EB3, four cells were imaged for each illumination mode. EB3 comets were automatically detected and their average intensity through time measured. Both numerically as visually, the photobleaching was found to be similar to the results shown in Figure 2A\&B.

For the MV3 cells, at least seven cells were imaged in each mode and similar bleb and filopodia dynamics were observed. On the particular MV3 cells that is shown in Figure 2, three filopodia blurring events were observed in the sequential lattice mode and none in the simultaneous field synthesis mode. For the resolution measurements on the clathrin-coated pits, we imaged five different cells for lattice and field synthesis. On the two cells we selected for visualization, we measured 10 well isolated pits for each imaging mode and computed the mean and the standard deviation of the full width half maximum in the axial direction. On the other cells, we manually measured axial profiles with ImageJ and found similar values as reported in supplementary figure S10.

\section{Data Availability}

The datasets acquired for this study are available from the corresponding author upon request.

\section{Code Availability}

The Matlab code is available under https://github.com/AdvancedImagingUTSW/

FieldSynthesis The instrument control software can be requested for academic use from the 
corresponding authors and will be delivered under material transfer agreements with HHMI and UT Southwestern Medical Center.

\section{Supplementary Material}

Refer to Web version on PubMed Central for supplementary material.

\section{Acknowledgements}

We would like to thank the Cancer Prevention Research Institute of Texas (RR160057 to R.F.), the National Institutes of Health (T32CA080621 to M.K., F32GM117793 to K.M.D., K25CA204526 to E.S.W.), and Human Frontier Science Program (LT000954/2015 to P.R.). We would also like to thank Khuloud Jaqaman (CPRIT R1216, UT Southwestern Endowed Scholars Program to K.J.) and Robert D. Goldman (R01GM106023) for supporting M.K. We also would like to thank Marcel Mettlen for preparing the SK-Mel2 cells.

\section{References}

1. Davidovits P \& Egger MD Scanning Laser Microscope. Nature 223, 831-831 (1969). [PubMed: 5799022]

2. Laissue PP, Alghamdi RA, Tomancak P, Reynaud EG \& Shroff H Assessing phototoxicity in live fluorescence imaging. Nature Methods 14, 657-661 (2017). [PubMed: 28661494]

3. Cranfill PJ et al. Quantitative assessment of fluorescent proteins. Nature Methods 13, 557-562 (2016). [PubMed: 27240257]

4. Stelzer EHK Light-sheet fluorescence microscopy for quantitative biology. Nature Methods 12, 2326 (2015). [PubMed: 25549266]

5. Chen B-C et al. Lattice light-sheet microscopy: Imaging molecules to embryos at high spatiotemporal resolution. Science 346 (2014).

6. Keller PJ, Schmidt AD, Wittbrodt J \& Stelzer EH Reconstruction of zebrafish early embryonic development by scanned light sheet microscopy. Science 322, 1065-1069 (2008). [PubMed: 18845710]

7. Hanser BM, Gustafsson MG, Agard DA \& Sedat JW Phase-retrieved pupil functions in wide-field fluorescence microscopy. J Microsc 216, 32-48 (2004). [PubMed: 15369481]

8. Zhao T et al. Multicolor 4D Fluorescence Microscopy using Ultrathin Bessel Light Sheets. Sci Rep 6, 26159 (2016). [PubMed: 27189786]

9. Gao L et al. Noninvasive Imaging beyond the Diffraction Limit of 3D Dynamics in Thickly Fluorescent Specimens. Cell 151, 1370-1385 (2012). [PubMed: 23217717]

10. Planchon TA et al. Rapid three-dimensional isotropic imaging of living cells using Bessel beam plane illumination. Nature Methods 8, 417-423 (2011). [PubMed: 21378978]

11. Vettenburg T et al. Light-sheet microscopy using an Airy beam. Nature Methods 11, 541-544 (2014). [PubMed: 24705473]

12. Fahrbach FO, Gurchenkov V, Alessandri K, Nassoy P \& Rohrbach A Self-reconstructing sectioned Bessel beams offer submicron optical sectioning for large fields of view in light-sheet microscopy. Optics Express 21 (2013).

13. Quirin $\mathrm{S}$ et al. Calcium imaging of neural circuits with extended depth-of-field light-sheet microscopy. Optics Letters 41 (2016).

14. Gao L Extend the field of view of selective plan illumination microscopy by tiling the excitation light sheet. Optics Express 23 (2015).

15. Fiolka R, Stemmer A \& Belyaev Y Virtual slit scanning microscopy. Histochem Cell Biol 128, 499-505 (2007). [PubMed: 17891411]

16. Baumgart E \& Kubitscheck U Scanned light sheet microscopy with confocal slit detection. Opt Express 20, 21805-21814 (2012). [PubMed: 23037300] 
17. Dean KM, Roudot P, Welf ES, Danuser G \& Fiolka R Deconvolution-free Subcellular Imaging with Axially Swept Light Sheet Microscopy. Biophys J 108, 2807-2815 (2015). [PubMed: 26083920]

18. Valm AM et al. Applying systems-level spectral imaging and analysis to reveal the organelle interactome. Nature 546, 162-167 (2017). [PubMed: 28538724]

19. Chang BJ, Perez Meza VD \& Stelzer EHK csiLSFM combines light-sheet fluorescence microscopy and coherent structured illumination for a lateral resolution below $100 \mathrm{~nm}$. Proc Natl Acad Sci U S A 114, 4869-4874 (2017). [PubMed: 28438995]

20. Kner P, Chhun BB, Griffis ER, Winoto L \& Gustafsson MGL Super-resolution video microscopy of live cells by structured illumination. Nature Methods 6, 339-342 (2009). [PubMed: 19404253]

21. Huisken J \& Stainier DYR Even fluorescence excitation by multidirectional selective plane illumination microscopy (mSPIM). Optics Letters 32 (2007).

22. Linkert $\mathrm{M}$ et al. Metadata matters: access to image data in the real world. The Journal of Cell Biology 189, 777-782 (2010). [PubMed: 20513764]

23. Kirshner H, Aguet F, Sage D \& Unser M 3-D PSF fitting for fluorescence microscopy: implementation and localization application. J Microsc 249, 13-25 (2013). [PubMed: 23126323]

24. Schindelin J et al. Fiji: an open-source platform for biological-image analysis. Nat Methods 9 , 676-682 (2012). [PubMed: 22743772] 
a

Fourier

Real

me-Averaged

Space

Space Intensity
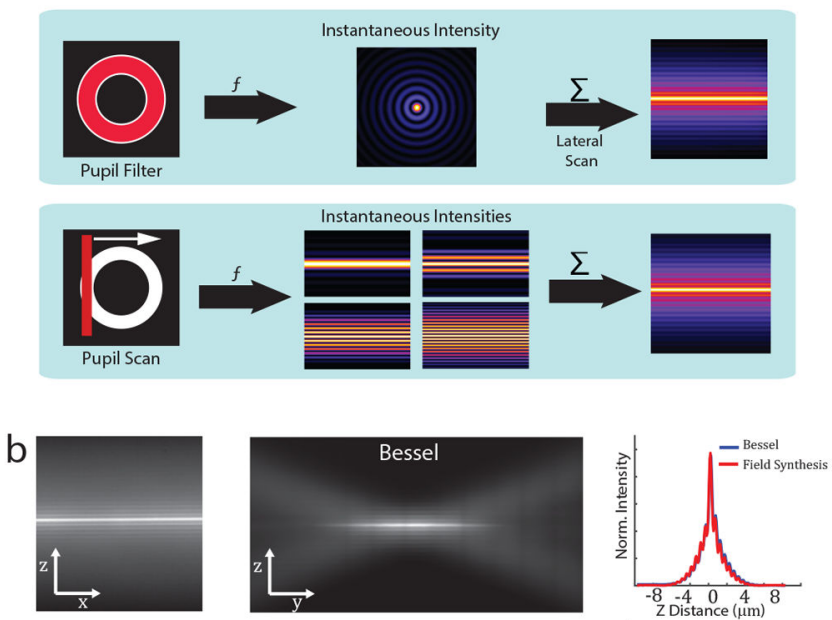

C
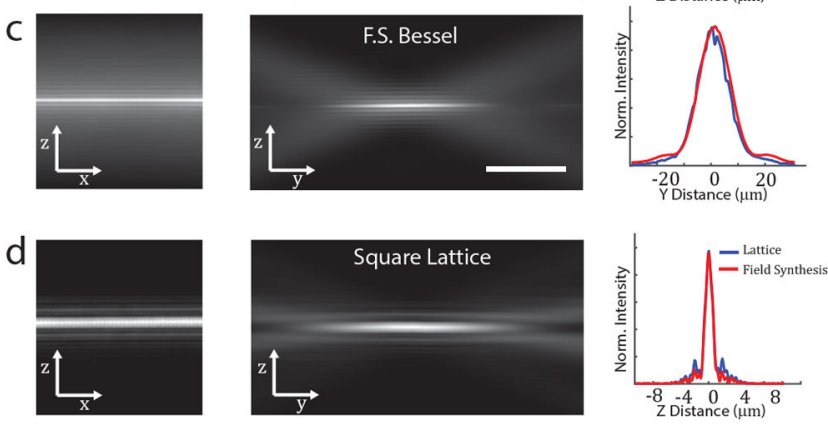

e
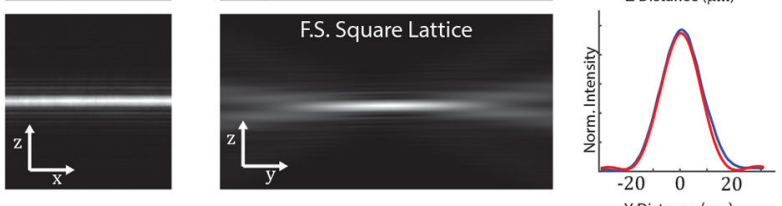

f

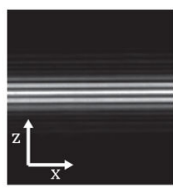

g
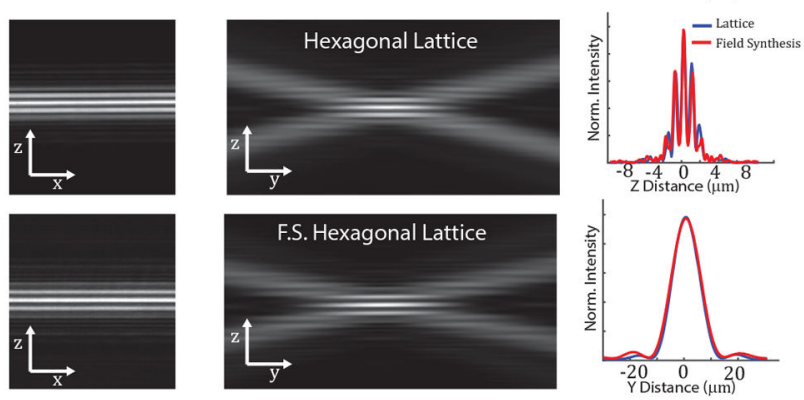

Figure 1.

Light-sheet generation by field synthesis. A, Top. In light-sheet microscopy, a pupil filter conjugate to the back-pupil plane of the illumination objective is used to shape the instantaneous laser focus at the front focal plane of the same objective. To generate a timeaveraged sheet of light, the laser focus is rapidly scanned laterally. A, Bottom. Principle of field synthesis: a focused line is laterally scanned over a pupil filter, creating a timeaveraged sheet of light in the front focal plane of the illumination objective. B, D, and F. Experimental examples of a Bessel beam light-sheet, square lattice, and hexagonal lattice, generated by traditional methods, respectively. C, E, and G. Experimental examples of a 
Bessel beam light-sheet, square lattice, and hexagonal lattice generated with Field Synthesis, respectively. The cross-sectional profiles are averaged over six spatial regions in each image dataset. 

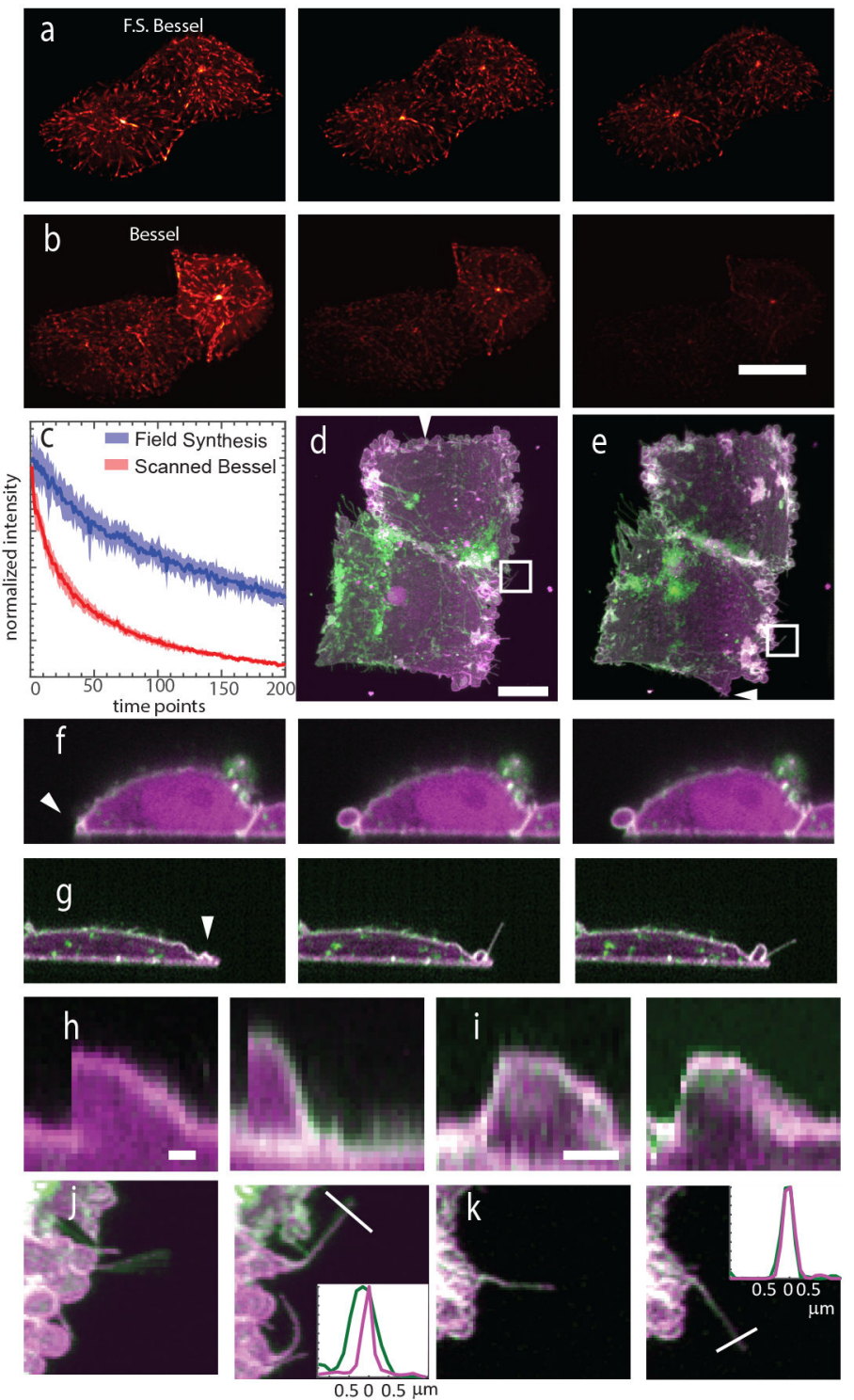

Figure 2.

Field Synthesis reduces photobleaching and enables simultaneous multicolor imaging. A.

Three timepoints (first, fifteenth, and thirtieth) of a movie of EB3 in an U2OS cell as imaged with Field Synthesis. B. Three time points (first, fifteenth, thirtieth) of a movie of EB3 in an U2OS cell as imaged with scanned Bessel Beam light-sheet microscopy. C. Comparison of photobleaching rate of 100nm fluorescent microspheres over 200 acquired volumes as imaged with Field Synthesis and Bessel Light-sheet microscopy. Shaded bands represent the $95^{\text {th }}$ percentile of each photobleaching curve, the solid line represents the mean (11 beads were measured for each condition). D MV3 cancer cell as imaged with sequential dual color square lattice light-sheet microscopy at a volumetric rate of $0.23 \mathrm{~Hz}$. Membrane labeled with td-Tomato-CCAX is shown in green and the GFP-Akt-PH biosensor is shown in magenta. E. Same cell imaged with Field Synthesis in square lattice mode at a volumetric rate of $0.47 \mathrm{~Hz}$. F. Three consecutive timepoints of a cross-section at position indicated by arrow in $\mathbf{D}$. G. Three consecutive timepoints of a cross-section indicated by an arrow in E. H. Kymographs 
of two blebs imaged with square lattice light-sheet microscopy. Onset of bleb formation is discontinuous. I. Kymographs of two blebs imaged with Field Synthesis. Growth of bleb is gradual due to increased sampling frequency. $\mathbf{J}$. Magnified view of the boxed region in $\mathbf{D}$ showing two timepoints of filopodia dynamics. K. Magnified view of the boxed region in $\mathbf{E}$ showing two timepoints of filopodia dynamics. Scale bar: A,B,D: 10 microns; H,I: 20s. Repeatability: A,B four cells were imaged in each light-sheet mode and a similar difference in photo-bleaching was observed. D-K: Seven cells were imaged in the lattice mode and 9 cells in the Field synthesis mode, yielding similar results. 